\title{
Metformin and mortality
}

\author{
S. S. Lund
}

Received: 28 November 2012 / Accepted: 30 December 2012 / Published online: 2 February 2013

(C) Springer-Verlag Berlin Heidelberg 2013

Keywords Metformin $\cdot$ Mortality $\cdot$ Randomised clinical trials · Type 2 diabetes mellitus

\begin{tabular}{|c|c|}
\hline \multicolumn{2}{|c|}{ Abbreviations } \\
\hline HOME & $\begin{array}{l}\text { Hyperinsulinaemia: the Outcome of its } \\
\text { Metabolic Effects }\end{array}$ \\
\hline $\mathrm{RCT}$ & Randomised clinical trial \\
\hline UKPDS & UK Prospective Diabetes Study \\
\hline
\end{tabular}

To the Editor: In an important and carefully performed metaanalysis of randomised clinical trials (RCTs) of metformin therapy in individuals with and without diabetes, Stevens and colleagues [1] showed a neutral effect of metformin vs comparator on cancer (RR 1.02; 95\% CI 0.82, 1.26) and on all-cause mortality (RR $0.99 ; 95 \%$ CI $0.83,1.17$ ). The authors included the two RCTs of metformin conducted by the UK Prospective Diabetes Study (UKPDS). These two RCTs, however, showed conflicting results of metformin vs comparator for all-cause mortality. One RCT by the UKPDS of metformin vs usual care (primarily diet alone) in overweight/obese patients with type 2 diabetes (termed UKPDS-O by Stevens and colleagues) showed an RR of 0.64 (95\% CI 0.45, 0.91), that is, a possible beneficial effect of metformin. In contrast, the other RCT by the UKPDS of metformin vs usual care in a mixed group of non-overweight and overweight/obese type 2 diabetic patients with sulfonylurea failure (termed UKPDS-S by Stevens and colleagues) showed an RR of 1.60 (95\% CI $1.02,2.52)$, that is, a possible harmful effect of metformin [1, 2]. In their meta-analysis, Stevens and colleagues performed a

\footnotetext{
S. S. Lund $(\bowtie)$

Frederiksborgvej 139 st. B,

2400 Copenhagen NV, Denmark

e-mail: sslund@dadlnet.dk
}

post hoc sensitivity analysis excluding the UKPDS-S, the study suggesting possible harm caused by metformin vs usual care. This sensitivity analysis showed a statistically significant reduction in all-cause mortality of metformin vs placebo/usual care (RR 0.67; 95\% CI 0.49, 0.93). However, Stevens and colleagues did not report a corresponding sensitivity analysis excluding the UKPDS-O, the study suggesting a possible benefit of metformin vs usual care. The authors' rationale for not reporting this analysis was not stated. Only these two studies - the UKPDS-O and UKPDS-S - showed statistically significant differences between metformin vs comparator in the meta-analysis by Stevens and colleagues, and the differences were only for all-cause mortality. The UKPDS-O had different targets for glucose control in the metformin and usual care arms (fasting plasma glucose of $<6.0 \mathrm{mmol} / 1$ and $<15 \mathrm{mmol} / \mathrm{l}$, respectively), whereas the UKPDS-S had similar targets for glucose control (fasting plasma glucose $<6.0 \mathrm{mmol} / \mathrm{l}$ ) in both arms [2]. It could therefore be argued that the UKPDS-S is the more 'fair' comparison of metformin against usual care of these two trials because it controls for glucose (targets) between the interventions. Also, performing a meta-analysis that considers only beneficial effects of the investigational intervention, e.g. performing sensitivity analyses excluding only results suggesting harm, e.g. UKPDS-S, as done by Stevens and colleagues, is, by nature, premature and may introduce bias into the interpretation. To facilitate a more balanced evidence-base and more balanced communication about the use of metformin, I hope Stevens and colleagues will provide the missing sensitivity analyses of their meta-analysis for all-cause mortality of metformin vs placebo/usual care and metformin vs any comparator, excluding the UKPDS-O study. In the past, various epidemiological considerations have been used to cast doubt on the validity of the results from the UKPDS-S [2-4], an RCT. In their discussion, Stevens and colleagues seem to somewhat favour these epidemiological views [1]. Data from 
epidemiological studies should, however, probably be considered less confirmatory than data from RCTs and it is unlikely that any level of epidemiological data can refute (or confirm) data from RCTs - only new RCTs may do so. Thus, strictly speaking, not even nearly 60 years of (non-randomised) clinical use of metformin - or, an ever increasing number of guidelines recommending more or less unrestricted use of metformin in all type 2 diabetic patients - can show that metformin is a safe drug. Thus, the question of whether the mortality results of either the UKPDS-O or UKPDS-S were down to chance can probably only be answered by new RCTs, and none appear to have been initiated to date. It has been suggested that metformin could potentially inhibit respiration in the mitochondria, the 'breathing lung' of the cell [5]. Some researchers even refer to metformin as being a 'mitochondrial poison' [6]. The potential long-term effects of such possible inhibition do not seem to be well understood, and mitochondrial dysfunction has been suggested to contribute to ageing and various chronic diseases, such as cardiovascular disease [7]. Thus, from a mitochondrial perspective, it could be argued that using metformin to promote health in humans could be counterproductive. This mechanism could underlie the adverse effect of metformin on mortality observed in the UKPDS-S. In turn, this further supports that possible harm, not only benefit, from metformin should be considered when designing trials or performing meta-analyses on this drug (not to mention making guidelines). Although a number of RCTs of metformin vs comparator have been conducted, some of which were included in the present meta-analysis by Stevens and colleagues [1], as the authors point out, the question of the impact of long-term metformin treatment, if any, on patientrelevant outcomes (e.g. vascular disease, cancer and mortality) in diverse populations remains to be established by adequately designed RCTs. For example, despite the results reported in the present meta-analysis, without other adequately designed RCTs refuting the adverse findings from UKPDS$\mathrm{S}$, it remains to be established that harm (beyond the wellknown rare events of lactic acidosis) can generally be assumed to be an unlikely event associated with metformin treatment. Stevens and colleagues did not include the Hyperinsulinaemia: the Outcome of its Metabolic Effects (HOME) trial in their meta-analysis, probably because the sample size in HOME was below that stated as an inclusion criterion of the meta-analysis of at least 500 participants. In HOME, 390 type
2 diabetic patients receiving insulin therapy were randomised to receive metformin or placebo over 4 years, with vascular events and mortality reported as the primary outcome [8]. That is, HOME was a long-term RCT of metformin vs placebo with hard clinical endpoints as the primary outcome. Encouragingly, a low number of deaths were observed in HOME. However, it may still be noted that, nominally, in HOME, all-cause mortality was not in favour of metformin vs placebo $(n=9$ [4.6\%] vs $n=6[3.1 \%]$, respectively; statistical test not reported) [8]. This probably also supports the need to further explore and understand the effect on mortality of metformin treatment in diverse populations, be it neutral, beneficial or harmful.

Duality of interest SSL is an employee of Boehringer Ingelheim (Ingelheim, Germany) and reports having stocks in Novo Nordisk A/S .

Contribution statement This article represents the author's personal opinion only, not the opinion of any third party. SSL was responsible for the conception, design and drafting of the manuscript, and approved the final version for publication.

\section{References}

1. Stevens RJ, Ali R, Bankhead CR et al (2012) Cancer outcomes and all-cause mortality in adults allocated to metformin: systematic review and collaborative meta-analysis of randomised clinical trials. Diabetologia 55:2593-2603, Erratum 55:3399-3400

2. UK Prospective Diabetes Study (UKPDS) Group (1998) Effect of intensive blood-glucose control with metformin on complications in overweight patients with type 2 diabetes (UKPDS 34). Lancet 352:854-865, Erratum 352:1558

3. Evans M (1998) The UK Prospective Diabetes Study. Lancet 352: 1932-1933

4. Turner RC, Holman RR (1999) Metformin and risk of cardiovascular disease. Cardiology 91:203-204

5. Hardie D, Ross F, Hawley S (2012) AMP-Activated protein kinase: a target for drugs both ancient and modern. Chem Biol 19:1222-1236

6. Corominas-Faja B, Quirantes-Pine R, Oliveras-Ferraros $\mathrm{C}$ et al (2012) Metabolomic fingerprint reveals that metformin impairs one-carbon metabolism in a manner similar to the antifolate class of chemotherapy drugs. Aging (Albany NY) 4:480-498

7. Dai DF, Rabinovitch PS, Ungvari Z (2012) Mitochondria and cardiovascular aging. Circ Res 110:1109-1124

8. Kooy A, de Jager J, Lehert P et al (2009) Long-term effects of metformin on metabolism and microvascular and macrovascular disease in patients with type 2 diabetes mellitus. Arch Intern Med 169:616-625 